\title{
Influence of Low FODMAP and Gluten-Free Diets on Gut Microbiota Alternations and Symptom Severity in Iranian Patients With Irritable Bowel Syndrome; A Clinical Trial Study
}

\section{Kaveh Naseri}

Department of Microbiology, School of Medicine, Shahid Beheshti University of Medical Sciences, Tehran, Iran

Hossein Dabiri

Department of Microbiology, School of Medicine, Shahid Beheshti University of Medical Sciences, Tehran, Iran

\section{Mohammad Rostami Nejad ( $\nabla$ m.rostamii@gmail.com )}

Celiac Disease Department, Gastroenterology and Liver Diseases Research Center, Research Institute for Gastroenterology and Liver Diseases, Shahid Beheshti University of Medical Sciences, Tehran, Iran

\section{Abbas Yadegar}

Foodborne and Waterborne Diseases Research Center, Research Institute for Gastroenterology and Liver Diseases, Shahid Beheshti University of Medical Sciences, Tehran, Iran

\section{Hamidreza Houri}

Foodborne and Waterborne Diseases Research Center, Research Institute for Gastroenterology and Liver Diseases, Shahid Beheshti University of Medical Sciences, Tehran, Iran

\section{Meysam Olfatifar}

Celiac Disease Department, Gastroenterology and Liver Diseases Research Center, Research Institute for Gastroenterology and Liver Diseases, Shahid Beheshti University of Medical Sciences, Tehran, Iran

\section{Amir Sadeghi}

Celiac Disease Department, Gastroenterology and Liver Diseases Research Center, Research Institute for Gastroenterology and Liver Diseases, Shahid Beheshti University of Medical Sciences, Tehran, Iran

\section{Saeede Saadati}

Celiac Disease Department, Gastroenterology and Liver Diseases Research Center, Research Institute for Gastroenterology and Liver Diseases, Shahid Beheshti University of Medical Sciences, Tehran, Iran

\section{Carolina Ciacci}

Gastrointestinal Unit, Department of Medicine, Surgery and Dentistry Scuola Medica Salernitana, Università di Salerno, Via Allende, 84081 Salerno, Italy

\section{Paola lovino}

Gastrointestinal Unit, Department of Medicine, Surgery and Dentistry Scuola Medica Salernitana, Università di Salerno, Via Allende, 84081 Salerno, Italy 


\section{Mohammad Reza Zali}

Celiac Disease Department, Gastroenterology and Liver Diseases Research Center, Research Institute for Gastroenterology and Liver Diseases, Shahid Beheshti University of Medical Sciences, Tehran, Iran

\section{Research Article}

Keywords: Irritable bowel syndrome, gluten-free diet, low-FODMAP, gut microbiota, IBS-SSS, Iran

Posted Date: February 6th, 2021

DOl: https://doi.org/10.21203/rs.3.rs-136688/v1

License: (c) (1) This work is licensed under a Creative Commons Attribution 4.0 International License. Read Full License

Version of Record: A version of this preprint was published at BMC Gastroenterology on July 14th, 2021. See the published version at https://doi.org/10.1186/s12876-021-01868-5. 


\section{Abstract}

Background \& objective: Recently, dietary restriction of fermentable carbohydrates (a low-FODMAP diet) in combination with a gluten-free diet (GFD) has been proposed to reduce the symptoms in irritable bowel syndrome (IBS) patients. Different studies reported that IBS has been associated with dysbiosis in the gut microbiota. Additionally, a few studies have reported inflammation in gastrointestinal (GI) system of adults with IBS. In this study we aim to investigate the effects of GFD and low-FODMAP diet on clinical symptoms, intestinal microbiota diversity, and fecal calprotectin (FC) level in Iranian patients with IBS.

Design: In this clinical trial study, 42 patients with IBS (Rome IV criteria) underwent GFD + low-FODMAP dietary intervention for 6 weeks. Symptoms were assessed using the IBS symptom severity scoring (IBSSSS), and fecal samples were collected at baseline and after intervention and analyzed by quantitative 16S rRNA PCR assay. The diversity of gut microbiota compared before and after 6 weeks of dietary intervention. FC was also analyzed by ELISA method.

Results: Thirty patients (mean age $37.8 \pm 10.7$ years) completed the 6-week diet. The IBS-SSS was significantly $(P=0.038)$ reduced after GFD + low-FODMAP dietary intervention compared to the baseline. Significant microbial differences before and after intervention were noticed in fecal samples. A significant increase was found in Bacteroidetes, and the Firmicutes to Bacteroidetes (F/B) ratio was significantly ( $P$ $=0.001)$ decreased after the dietary intervention. The value of FC was significantly decreased after 6 weeks dietary intervention $(P=0.001)$.

Conclusions: Our study suggests that patients with IBS under a GFD + low-FODMAP diet had a significant improvement in IBS symptoms severity, with reduced FC level following normalization of their gut microbiota composition. Further rigorous trials are needed to establish long-term efficacy and safety of this dietary intervention for personalized nutrition in IBS.

\section{Introduction}

Irritable bowel syndrome (IBS) is one of the most commonly diagnosed functional gastrointestinal disorders accounting for around $10 \%$ of the world's population [1]. This functional bowel disorder can be classified into different categories: diarrhea-predominant (IBS-D), constipation-predominant (IBS-C), mixed (IBS-M) and unclassified (IBS-U) [2].

The pathogenesis of IBS is complex and not yet clearly defined, but numerous pathophysiological mechanisms have been proposed including brain-gut dysfunction, intestinal dysmotility, visceral hypersensitivity, inflammation, psychosocial stressors, altered levels of gastrointestinal neuropeptides and hormones, and particularly imbalance in the composition of gut microbiota, called "microbiome dysbiosis" [3-5]. It is now well evident that microbiome dysbiosis-related disorders and IBS have been reported to have similar clinical features, and more recently, IBS has been found to be in association with the bacterial community shift in the large intestine [6, 7]. Furthermore, restoration and modulation of gut microbiota through consumption of probiotics, prebiotics, and symbiotic, which result in improvement of 
the IBS-related symptoms highlights the important role of gut microbiota dysbiosis in the pathogenesis of IBS [8-10].

Normal gut microbiome inhibits the overgrowth and colonization of pathogenic organisms by competition for nutrients and attachment sites in the gut epithelium, production of antibacterial substances, as well as enhancement of the host immune responses [11, 12]. Several previous studies have described that alterations in the gut microbiota such as increased number of Enterobacteriaceae and Bacteroidetes or decreased number of beneficial organisms like Bifidobacterium and Lactobacillaceae, can trigger gut immune response, impair gastrointestinal functions and enhance disease susceptibility $[9,13,14]$.

A majority of IBS patients suffer from abdominal discomfort or pain and bloating after ingestion of certain food items that may contain IBS-associated triggers $[15,16]$. In addition, a number of studies suggest that environmental factors such as diet, lifestyle, and antibiotics and medications exert a significant impact on the gut microbiome [17]. Recently, dietary components including wheat, gluten and fermentable oligo-di-mono-saccharides and polyols (FODMAP) has been suggested to play an essential role in induction of IBS symptoms [18]. Accordingly, there are some evidence supporting a clinically relevant positive effect for low-FODMAP and gluten-free diets in patients with IBS [19].

The significant effect acknowledged to date is alternation in gut microbiota by varying gluten intake, such as altering total microbiota abundance and changing the relative amount of Bifidobacteria [16, 20]. However, the roles of alterations of gut microbiota due to the reduction in FODMAP intake in ongoing efficacy have yet to be explored. Herein, we aimed to investigate the impact of a 6-week gluten-free diet (GFD) + low FODMAP diet on gut microbiota alterations in Iranian patients with IBS. Additionally, in order to determine the inflammation status of patients, we examined the level of fecal calprotectin (FC) before and after dietary intervention.

\section{Materials And Methods}

\section{Study population}

Ninety-six consecutive IBS patients were screened for eligibility; 54 patients did not meet the initial assessment criteria, and 12 patients discontinued the dietary intervention during the follow-up phase and 30 patients completed the study. A flowchart of the recruitment process and study design is shown in Figure 1. Forty-two eligible patients with IBS, aged 18-59 years (mean age $37.8 \pm 10.7$ years), and had a physician diagnosis of IBS according to the Rome IV diagnostic criteria, and did not have any other gastrointestinal disorders were recruited in this study [21]. Patients with following criteria were excluded: patients with history of celiac disease (CD), inflammatory bowel disease (IBD), liver diseases, gastrointestinal surgery, cancer, use of non-steroidal anti-inflammatory drugs (NSAIDs), excessive alcohol consumption, systemic use of immunosuppressive agents, and poorly controlled psychiatric disease. In addition, colonoscopy and biopsy specimens were performed in order to include patients with normal mucosa and excluding those with fissures, hemorrhoid, and microscopic colitis. Moreover, those who 
used broad-spectrum antibiotics, probiotics, or any other drugs that affect the bowel function within the last 4 weeks prior to study were also excluded. Active participation in another form of dietary therapy at the time of enrollment (i.e., low carbohydrate, high protein) was not considered in this study.

\section{Study design and procedures}

This study was an uncontrolled, open-label clinical trial study, which was conducted in the Research Institute for Gastroenterology and Liver Diseases (RIGLD) at Taleghani Hospital in Tehran, Iran. All the participants were recruited between March 2018 and August 2019. This was a dietary trial and the dietitians were not blinded. Patients referred to the trained research dietitian at the RIGLD and were counseled on their allocated diets. A standardized questionnaire including demographic information, usual diet, medication history, and underlying health condition and clinical symptoms was recorded for all patients. All IBS patients fulfilling inclusion criteria were counseled by the dietitian and given a GFD + low-FODMAP diet for a 6-week run-in period. Fecal samples were collected from all patients before and after completion of the study period for microbiota analysis. In order to assess the effect of the dietary interventions and the severity of IBS symptoms, the IBS symptom severity score (IBS-SSS) was applied [22]. The overall IBS-SSS ranges from 0 to 500, and all patients were divided into 3 severity groups by use of the accepted cut-off values: $<175$, mild IBS; $175-300$, moderate IBS; $>300$, severe IBS. A higher score implicates more severe symptoms. The study protocol was approved by the Ethical Review Committee of RIGLD at Shahid Beheshti University of Medical Sciences (Project No. IR.SBMU.RIGLD.REC.1396.154). The study was performed according to the revised Declaration of Helsinki 2013 [23] and informed consents were obtained from all subjects and/or their legal guardians prior to sample collection. This study was registered in Iranian Registry of Clinical Trials in 20/10/2018 and the clinical trial registration number is IRCT20100524004010N26.

\section{Collection of fecal samples}

Fresh stool samples were collected from every subject enrolled in this study at baseline and after 6 weeks following dietary therapy. All samples were homogenized thorough agitation using a vortex and divided into three aliquots within $3 \mathrm{~h}$ of defecation. The aliquots were immediately frozen and stored at $-80^{\circ} \mathrm{C}$ in screw-capped cryovial tubes until used for DNA extraction.

\section{DNA extraction from fecal samples}

Total DNA content was extracted from stool samples using the QIAamp DNA Stool Mini Kit (Qiagen Retsch $\mathrm{GmbH}$, Hannover, Germany) according to the manufacturer's instructions with some modifications. DNA concentration was quantified by NanoDrop ND-2000 Spectrophotometer (NanoDrop products, Wilmington, DE, USA). The concentration and purity of extracted DNA were assessed by Nanodrop (DeNovix Inc., USA). Extracted DNA samples were stored at $-20^{\circ} \mathrm{C}$ until further analysis.

\section{Microbiota analysis by quantitative real-time PCR (qPCR)}


In the current study, a qPCR assay was performed for the enumeration of eight bacterial phyla, families, and genera including Firmicutes, Bacteroidetes, Actinobacteria, Enterobacteriaceae, Bifidobacterium, Lactobacillus, Ruminococcus, and Streptococcus. The qPCR was carried out by SYBR Green chemistry using universal and group-specific primers based on the bacterial 16S rRNA sequences presented in Table S1. Each PCR reaction was performed in a final volume of $25 \mu \mathrm{L}$, comprising of $12.5 \mu \mathrm{L}$ of SYBR green PCR master mix (Ampliqon, Odense, Denmark), $1 \mu \mathrm{L}$ of $10 \mathrm{pmol}$ of forward and reverse primers, and 100 ng of the DNA template. The reaction parameters for amplification were $95^{\circ} \mathrm{C}$ for $10 \mathrm{~min}$ and 40 cycles at $95^{\circ} \mathrm{C}$ for $20 \mathrm{~s}, 30 \mathrm{~s}$ of annealing at optimal temperature for each primer pair as indicated in Table S1, and $72^{\circ} \mathrm{C}$ for 20 s. All PCR amplifications were carried out in triplicate by using a Rotor-Gene® Q (Qiagen, Germany) real-time PCR system. The accuracy of amplification was determined by melting curve analysis with increasing temperature from 60 to $95^{\circ} \mathrm{C}$ (at regular increment of $0.5^{\circ} \mathrm{C}$ for $5 \mathrm{~s}$ ) to confirm the specificity of amplification. The relative abundance of each taxon before and after the dietary therapy was calculated according to the ratio of 16S rRNA copy number of specific bacteria to total 16S rRNA copy number of universal bacteria using the previously described method [24]. Accordingly, the average $\mathrm{Ct}$ value obtained from each primer pair was transformed into a percentage using the following formula:

$$
X=\frac{\text { (Eff.Univ) }^{\text {ct univ }}}{\text { (Eff.Spec }^{\text {ct } \operatorname{spec}}} \times 100
$$

The "Eff.Univ" refers to the calculated efficiency of the universal primers $(2=100 \%$ and $1=0 \%)$ and "Eff.Spec" indicates the efficiency of the taxon-specific primers. "Ct univ" and "Ct spec" represent the threshold cycles registered by the thermocycler. " $X$ " represents the percentage (\%) of 16S taxon-specific copy number existing in a sample.

\section{Fecal calprotectin measurement}

Before and after the dietary therapy, first morning stool samples were collected from patients and stored at $-80^{\circ} \mathrm{C}$ after initial storage. $\mathrm{FC}$ was measured by using BÜHLMANN fCAL ${ }^{\circledR}$ ELISA kit (Bühlmann Laboratories, Schönenbuch, Switzerland) according to the manufacturer's instructions. A FC level $<50$ $\mu \mathrm{g} / \mathrm{g}$ stool was considered to be within the normal range.

\section{Statistical analysis}

Spearman's correlation analysis was used for non-parametric values and student $t$ test and MannWhitney test were used for analysis of parametric data. The PCA (principle component analysis) plot was drown by using the FactoMineR and Factoextra packages from the open source statistical program $\mathrm{R}$ version 3.6.1 ( $R$ Core Team, Vienna, Austria). The relative abundance of microbiota was graphed using GraphPad Prism software version 8.3.0 (GraphPad software, San Diego, CA, USA). Differences were considered to be statistically significant when $* P<0.05$, $* \star P<0.01$, and $* \star \star P<0.001$.

\section{Results}




\section{Baseline characteristics}

Out of 96 IBS patients, 30 patients completed the study period (a six-week of GFD + low-FODMAP diet), of whom 15 (50\%) patients were male, mean age 35.7 years (range 21 to 57 years), and 15 patients (50\%) were female, mean age 39.8 years (range 18 to 59 years). All IBS patients were sub-classified into IBS-D according to the Rome IV criteria. (Table 1).

Table 1

Baseline characteristics of study participants at enrollment.

\begin{tabular}{|ll|}
\hline \multicolumn{2}{|l|}{ Baseline characteristics of the participants } \\
\hline Age (years), mean (range) & $37.8(18-59)$ \\
\hline Gender (M/F) & $15 / 15$ \\
\hline Smoking, yes (\%) & $9(30 \%)$ \\
\hline IBS-SSS, mean (range) & $326(250-475)$ \\
\hline Ethnicity & \\
Persian & 10 \\
Turk & 11 \\
Kurd & 4 \\
Lur & 3 \\
Others & 2 \\
\hline
\end{tabular}

\section{Effects of the GFD + low-FODMAP diet on the microbiota diversity}

Our intestinal microbiota analysis was based on qPCR amplifications to calculate the ratio of 16S rRNA copy number of each bacterial taxa before and after the dietary intervention. The relative abundance and diversity of intestinal microbiota among IBS patients before and after the GFD and low-FODMAP dietary intervention is illustrated in Fig. 2A and 2B. The predominant phylum in IBS patients before the GFD + low-FODMAPs diet was Firmicutes (31.59\%), which significantly reduced after the dietary intervention (22.17\%; $P=0.003)$. Bacteroidetes was significantly the most abundant phylum after the dietary intervention, (from $11.69 \%$ (baseline) to $26.65 \% ; P=0.001$ ). The relative abundance of phylum Actinobacteria was also increased after the dietary intervention, but this difference was not statistically significant $(P=0.12)$. No significant $(P=0.63)$ alterations were also observed in relative abundance of Enterobacteriaceae before and after the intervention. However, the percentage of Ruminococcaceae significantly decreased from 6.4 to $3.45 \%(P=0.001)$. At the genus level, the relative abundance of Bifidobacterium and Lactobacillus significantly $(P=0.001$ and $P=0.006$, respectively) increased after the intervention, whereas the percentage of Streptococcus remained almost similar to its baseline abundance with no significant changes $(P=0.72)$. The mean percentage and distribution of the selected bacterial taxa before and after GFD + low-FODMAP diet in IBS patients are illustrated in Figs. 3 and 4. In addition, 
principal component analysis (PCA) also revealed that taxonomic profiles were notably different in the microbial communities before and after the dietary intervention among IBS patients as schematically depicted in Fig. 5. The ratio of Firmicutes to Bacteroidetes (F/B) was significantly decreased $(P=0.001)$ and shifted from 2.6:1 to $0.8: 1$ before and after the dietary intervention, respectively. The F/B ratio before and after GFD and low-FODMAP diet in IBS patients is shown in Fig. 6.

\section{Effects of the GFD + low-FODMAP diet on IBS symptom severity}

Figure 7 represents IBS symptom severity in each patient before and after the dietary intervention. Based on the IBS-SSS, the severity of IBS symptoms was classified as mild $(n=1)$, moderate $(n=12)$ and severe $(n=17)$ at the baseline and before the dietary intervention. After the end of the dietary intervention, the number of patients in each classification changed to mild $(n=8)$, moderate $(n=20)$ and severe $(n=2)$, accordingly (Table 2. Totally, IBS-SSS decreased in 22/30 (73.3\%) patients after the dietary intervention compared to the baseline, and this clinical improvement was statistically significant $(P=0.038)$.

Approximately, fifty-three percent of patients after the end of the dietary intervention experienced $30-60 \%$ reduction in IBS-SSS, while only $3.3 \%$ of patients of experienced more than $60 \%$ reduction (Table 3 ).

Table 2

Severity scores for mild, moderate and severe IBS patients before and after the dietary intervention.

\begin{tabular}{|c|c|c|c|c|c|c|c|}
\hline \multirow[t]{2}{*}{ IBS-SSS } & \multicolumn{3}{|l|}{ Before } & \multirow[t]{2}{*}{ IBS-SSS } & \multicolumn{3}{|l|}{ After } \\
\hline & Mean $( \pm S D)$ & Median & Range & & Mean ( \pm SD) & Median & Range \\
\hline $\begin{array}{l}\text { Mild } \\
(n=1)\end{array}$ & $175( \pm 0.0)$ & 175 & 175 & $\begin{array}{l}\text { Mild } \\
(\mathrm{n}=8)\end{array}$ & $146.8( \pm 19.5)$ & 150 & $\begin{array}{l}100- \\
175\end{array}$ \\
\hline $\begin{array}{l}\text { Moderate } \\
(n=12)\end{array}$ & $\begin{array}{l}277.5( \pm \\
23.4)\end{array}$ & 287.5 & $\begin{array}{l}250- \\
300\end{array}$ & $\begin{array}{l}\text { Moderate } \\
(n=20)\end{array}$ & $\begin{array}{l}231.25( \pm \\
28.6)\end{array}$ & 237.5 & $\begin{array}{l}200- \\
300\end{array}$ \\
\hline $\begin{array}{l}\text { Severe } \\
(n=17)\end{array}$ & $\begin{array}{l}369.1 \\
29.1)\end{array}$ & 350 & $\begin{array}{l}300- \\
450\end{array}$ & $\begin{array}{l}\text { Severe } \\
(n=2)\end{array}$ & $375( \pm 25)$ & 375 & $\begin{array}{l}350- \\
400\end{array}$ \\
\hline
\end{tabular}

Table 3

Frequency distribution of IBS-SSS reduction.

\begin{tabular}{|lll|}
\hline IBS-SSS reduction category & After dietary intervention $\mathbf{n}(\%)$ & $P$ value \\
\hline Decreased/Increased/unchanged & $22(73.3) / 2(6.6) / 6(20)$ & $<0.038$ \\
\hline$<30 \%$ reduction & $5(16.6)$ & \\
\hline $30-60 \%$ reduction & $16(53.3)$ & \\
\hline$>60 \%$ reduction & $1(3.3)$ & \\
\hline
\end{tabular}

\section{Fecal Calprotectin}


The mean FC values in the IBS patients at the baseline and after a 6-week gluten-free and low-FODMAP diet was shown in Figure 8. The value of FC was significantly decreased after 6 weeks diets, from 83.4 at baseline to $37.3(P<0.001)$. In addition, we did not observe any significant correlation between $\mathrm{FC}$ level and microbiota diversity before and after the diets.

\section{Discussion}

It has been hypothesized that a subset of patients with IBS may have an intolerance of dietary triggers such as gluten and highly FODMAP-containing foods, which can alter the gut microbiota and the metabolome of patients with IBS leading to worsening of their symptoms [25-27]. This has led researchers to recommend GFD and low-FODMAP diet as the most widely adopted diets to improve IBS symptoms [28-30]. Despite the growing popularity of the GFD and low-FODMAP diet in patients with IBS, the beneficial impact of such dietary interventions on restoration of intestinal microbiota dysbiosis has been lacking in this population.

In this 6-week, controlled, dietary intervention study, a clinically significant improvement in IBS-SSS was observed after the dietary intervention compared to the baseline. Several other clinical trials have also shown that a low-FODMAP diet associates with an improvement in IBS symptom scores and effectively increased quality of life in patients with IBS [19, 27, 31-34]. It is now well documented that IBS is a condition in which several pathophysiological mechanisms are involved in its development and symptom severity. Among them, a distinct fecal microbiota composition and microbiome dysbiosis has been proposed as one of the key factors associated with the disease symptom severity [14, 35, 36]. Data obtained from a recent systematic review demonstrated that phylum Proteobacteria, phylum Bacteroidetes, family Enterobacteriaceae, and family Lactobacillaceae increased in patients with IBS [14]. On the other hand, it was also documented that uncultured Clostridiales I, genus Faecalibacterium (including Faecalibacterium prausnitzii), and genus Bifidobacterium were decreased in patients with IBS. Moreover, it was revealed that the overall microbiota diversity was either decreased or not changed in IBS patients in comparison with healthy controls [14]. However, conflicting results of cumulative evidence for Firmicutes, Bacteroidetes, and Actinobacteria were observed among different studies [37-39].

There is increasing evidence that dietary interventions using low-FODMAP diets could improve functional gastrointestinal symptoms in IBS patients particularly through interactions with the gut microbiota [15, $19,40]$. Furthermore, another study suggested that reduction of FODMAPs intake in a GFD consistently and significantly improved the gastrointestinal symptoms in IBS patients who were finally classified as non-celiac gluten sensitivity (NCGS) [41]. In our study, we found that the relative abundance of phylum Firmicutes were higher prior to the start of dietary interventions compared to the phylum Bacteroidetes in patients with IBS. However, there was a clear tendency to increased Bacteroidetes after the dietary interventions, and subsequently the F/B ratio was significantly decreased. In a recent study by Dieterich et al., clinical and neurological symptoms of NCGS patients who consumed low-FODMAP diet and especially the GFD significantly improved [42]. In addition, they reported a significant increase in the numbers of Bacteroidetes following a 2-week GFD compared to the low-FODMAP diet $(P<0.01)$. 
Furthermore, data obtained from a randomized clinical trial in childhood IBS demonstrated that individuals who respond to a low-FODMAP diet have a greater capacity for saccharolytic metabolism mainly due to higher proportions of Bacteroidaceae, Erysipilotrichaceae and Clostridiales species than non- responders [33]. Rajilić-Stojanović et al. also reported an approximately 2-fold increase in the F/B ratio as the major bacterial phyla in 62 IBS patients (Rome II criteria) compared with 46 healthy subjects [43]. This finding has been observed in several other studies, in which the abundance of Firmicutes was enriched together with a reduced abundance of Bacteroidetes in the IBS subjects compared to healthy individuals [44-46]. In contrast, other studies reported an increase in the content and abundance of Bacteroidetes members in the IBS patients compared to non-IBS subjects $[47,48]$. Bacteroidetes are known as complex carbohydrate digesters which are specialized in degrading specific types of dietary fibers in order to maximize energy intake from these kinds of carbohydrates $[49,50]$.

The phylum Actinobacteria is proportionally less abundant phyla (3\%) of the human gut microbiota, and notably represented by the probiotic containing genera such as Bifidobacterium and Collinsella [36, 51]. Several studies have reported a significant depletion in Actinobacteria in the gut of patients with IBS [43, $45,52]$. On the other hand, other studies reported an increase in the relative abundance of Actinobacteria among IBS patients compared to healthy controls [39,44,53]. In our study the relative abundance of Actinobacteria was increased, although not statistically significant, after the GFD and low-FODMAP dietary intervention. In line with our results, Mclntosh et al. also reported that a 3-week low-FODMAP diet increased Actinobacteria richness and diversity in patients with IBS [27]. In contrast, a recent study had demonstrated that gut bacteria such as Actinobacteria, Bifidobacterium, and Faecalibacterium prausnitzii were significantly decreased in IBS patients throughout a 9-week low-FODMAP diet along with reduction in total SCFAs, n-butyric acid, and serum levels of proinflammatory cytokines (IL-6 and IL-8) as compared to baseline [54]. However, the overall inconsistency and differences in the abovementioned results contribute to the difference among study populations, IBS subtypes, duration of dietary intervention, level of dietary adherence, washout period in crossover studies, and variations in taste or other contents outside of FODMAP carbohydrates that may affect the dysbiotic gut microbiota and disease outcomes.

In fact, the majority of studies on gut microbiota analysis in IBS patients that used either q-PCR or nonquantitative methods reported a decrease in the abundance of Bifidobacterium and Lactobacillus $[55,56]$. However, Maccaferri et al. exceptionally reported an increase in the relative abundance of Lactobacilliand Bifidobacteria among IBS subjects [57]. A number of studies have shown that the low-FOMAPs diet decreases the abundance of Bifidobacterium in the gut of IBS patients [16, 20, 27, 31, 34, 54]. Collectively, previous studies have also demonstrated that GFD induced a decrease in Bifidobacterium spp. in the intestinal microbiome of healthy human subjects, celiac disease (CD) and NCGS patients, raising potential concerns after the consumption of a GFD $[42,58,59]$. However, Collado et al. reported increased prevalence of certain Bifidobacterium species including B. adolescentis, B. lactis and B. dentium after a GFD in CD subjects [60]. In another study from Brazil, the fecal counts of Bifidobacteria were significantly higher in GFD treated-CD (T-CD) patients compared to the healthy subjects $[59,61]$. Interestingly, we observed that the relative abundances of lactate-producing bacteria Bifidobacterium and Lactobacillus were increased after the GFD and low-FODMAP dietary intervention in IBS patients. To our knowledge, no 
previous studies have evaluated effects of the GFD and low-FODMAP dietary intervention simultaneously on IBS patients. Our findings propose that at least in patients with IBS implementing simultaneous GFD and low-FODMAP diet may lead to an increase in the relative abundances of Bifidobacterium and Lactobacillus, although contrary to the other studies which applied these dietary interventions separately. Therefore, it is noteworthy to administrate the diet or a combination of diets of choice for gut microbiotaassociated disorders such as IBS in order to normalize the dysbiotic communities of microbiota.

The family Enterobacteriaceae (phylum Proteobacteria), contains several pathogenic genera such as Escherichia, Shigella, Salmonella, and Campylobacter. Generally, these aerobes were found to be slightly enriched in IBS patients and significantly correlated with IBS symptoms. Moreover, an increase in some of these pathogenic microbiota may contribute to the low rate of mucosal inflammation through overexpression of proinflammatory cytokines IL- 6 and IL-8 as seen in IBS patients $[14,43,57,62]$. In contrast, Tana et al. found no difference in Enterobacteriaceae count between IBS patients and healthy controls. In another study by De Palma et al., Enterobacteriaceae were increased in a group of healthy adult subjects who were on a one-month GFD [63]. In our study, we found no difference in the relative abundance of Enterobacteriaceae before and after the dietary intervention.

The genus Streptococcus is among the dominant bacterial groups present in the upper gastrointestinal tract [64]. Some reports have also showed high fecal amounts of Streptococcus spp., pathogenic bacteria which causes increased expression levels of IL-6 [65], in IBS patients [43], and particularly in IBS-D subtypes $[46,66]$. We found no difference in relative abundance of Streptococcus after the dietary intervention compared to the baseline. Our results are in agreement with another study which found no difference in relative abundance of Streptococcus species for the low FODMAP diet compared with sham diet [31]. Furthermore, Bennet et al. also demonstrated no difference in abundance of Streptococcus between responders and non-responders patients with IBS after a 4-week low-FODMAP diet [34].

Our data suggest that there is a correlation between consuming a low-FODMAP diet combined with GFD and decrease of FC which is in line with a study performed by Shulman et al. [67], but are in contrast with a few previous studies which suggested that FC concentration was not increased in IBS patients compared with control subjects [68-70]. However, these controversial findings in the above-mentioned studies may be due to differences in the study design and population.

\section{Conclusion}

In summary, our study suggests that patients with IBS who consumed a low-FODMAP diet combined with GFD had a significant improvement in IBS symptoms, and normalization of their gut microbiota. In addition, our findings indicate inflammation in IBS patients. In which consuming a low-FODMAP diet combined with GFD can downregulate intestinal inflammation, and consequently decrease IBS-SSS. To the best of our knowledge, there has been no comprehensive analysis of the effects of a GFD and lowFODMAP diet on the gut microbiota in a group of unselected Iranian patients with IBS. We also suggest to evaluate the effects of this combined dietary intervention on the metabolic output of gut microbiota and 
its integration with supplementary probiotics to avoid side effects on health due to the unfavorable alteration of the intestinal microbiota in IBS individuals. Future studies are still required to validate the robustness of our findings, and to establish long-term efficacy and safety of this dietary intervention for personalized nutrition in IBS.

\section{Declarations}

\section{Ethics approval and consent to participate}

The study protocol was approved by the Ethical Review Committee of RIGLD at Shahid Beheshti University of Medical Sciences (Project No. IR.SBMU.RIGLD.REC.1396.154).

\section{Consent for publication}

Not applicable.

\section{Availability of data and materials}

The datasets supporting the conclusions of this article are included within the article and its additional file.

\section{Competing interests}

The authors declare that they have no conflicts of interest.

\section{Funding}

This study was supported by a grant (no. RIGLD 961) from Foodborne and Waterborne Diseases Research Center, Research Institute for Gastroenterology and Liver Diseases, Shahid Beheshti Statement of authors University of Medical Sciences, Tehran, Iran.

\section{Authors' contributions}

SS collected the samples and KN performed the real-time PCR analysis; MRN and AY designed and supervised the study; $\mathrm{KN}, \mathrm{AY}$, and $\mathrm{MO}$ participated in data analysis; $\mathrm{KN}, \mathrm{AY}$, and $\mathrm{HH}$ wrote the manuscript; MRN, AS, HD, CC, PI, and MRZ critically revised the manuscript. All authors approved the final version of the manuscript.

\section{Acknowledgements}

The authors wish to thank the laboratory staff of Foodborne and Waterborne Diseases Research Center, Research Institute for Gastroenterology and Liver Diseases, Shahid Beheshti University of Medical Sciences, Tehran, Iran. 


\section{References}

1. Lovell RM, Ford AC: Global prevalence of and risk factors for irritable bowel syndrome: a metaanalysis. Clinical gastroenterology and hepatology : the official clinical practice journal of the American Gastroenterological Association 2012, 10(7):712-721.e714.

2. Schmulson MJ, Drossman DA: What Is New in Rome IV. Journal of neurogastroenterology and motility 2017, 23(2):151-163.

3. Parkes GC, Brostoff J, Whelan K, Sanderson JD: Gastrointestinal microbiota in irritable bowel syndrome: their role in its pathogenesis and treatment. The American journal of gastroenterology 2008, 103(6):1557-1567.

4. Chey WD, Kurlander J, Eswaran S: Irritable Bowel Syndrome: A Clinical ReviewIrritable Bowel Syndromelrritable Bowel Syndrome. JAMA 2015, 313(9):949-958.

5. Javanmard A, Ashtari S, Sabet B, Davoodi SH, Rostami-Nejad M, Akbari ME, Niaz A, Mortazavian AM: Probiotics and their role in gastrointestinal cancers prevention and treatment; an overview. Gastroenterology and hepatology from bed to bench 2018, 11(4):284.

6. DuPont HL: Review article: evidence for the role of gut microbiota in irritable bowel syndrome and its potential influence on therapeutic targets. 2014, 39(10):1033-1042.

7. Holtmann G, Shah A, Morrison MJDD: Pathophysiology of functional gastrointestinal disorders: a holistic overview. 2017, 35(S1):5-13.

8. Pimentel M, Lembo A, Chey WD, Zakko S, Ringel Y, Yu J, Mareya SM, Shaw AL, Bortey E, Forbes WP: Rifaximin therapy for patients with irritable bowel syndrome without constipation. The New England journal of medicine 2011, 364(1):22-32.

9. Shukla R, Ghoshal U, Dhole TN, Ghoshal UC: Fecal Microbiota in Patients with Irritable Bowel Syndrome Compared with Healthy Controls Using Real-Time Polymerase Chain Reaction: An Evidence of Dysbiosis. Digestive diseases and sciences 2015, 60(10):2953-2962.

10. Rostami-Nejad M, Ishaq S, Al Dulaimi D, Zali MR, Rostami K: The role of infectious mediators and gut microbiome in the pathogenesis of celiac disease. Archives of Iranian Medicine 2015, 18(4):0-0.

11. Lee BJ, Bak YT: Irritable bowel syndrome, gut microbiota and probiotics. Journal of neurogastroenterology and motility 2011, 17(3):252-266.

12. Azimirad M, Rostami-Nejad M, Rostami K, Naji T, Zali MR: The susceptibility of celiac disease intestinal microbiota to Clostridium difficile infection. American Journal of Gastroenterology 2015, 110(12):1740-1741.

13. Ohman L, Simren M: Pathogenesis of IBS: role of inflammation, immunity and neuroimmune interactions. Nature reviews Gastroenterology \& hepatology 2010, 7(3):163-173.

14. Pittayanon R, Lau JT, Yuan Y, Leontiadis GI, Tse F, Surette M, Moayyedi P: Gut Microbiota in Patients With Irritable Bowel Syndrome-A Systematic Review. Gastroenterology 2019, 157(1):97-108.

15. Bohn L, Storsrud S, Liljebo T, Collin L, Lindfors P, Tornblom H, Simren M: Diet low in FODMAPs reduces symptoms of irritable bowel syndrome as well as traditional dietary advice: a randomized 
controlled trial. Gastroenterology 2015, 149(6):1399-1407.e1392.

16. Staudacher HM, Lomer MCE, Anderson JL, Barrett JS, Muir JG, Irving PM, Whelan K: Fermentable Carbohydrate Restriction Reduces Luminal Bifidobacteria and Gastrointestinal Symptoms in Patients with Irritable Bowel Syndrome. The Journal of Nutrition 2012, 142(8):1510-1518.

17. Chong PP, Chin VK, Looi CY, Wong WF, Madhavan P, Yong VC: The Microbiome and Irritable Bowel Syndrome - A Review on the Pathophysiology, Current Research and Future Therapy. Front Microbiol 2019, 10:1136-1136.

18. De Giorgio R, Volta U, Gibson PR: Sensitivity to wheat, gluten and FODMAPs in IBS: facts or fiction? Gut 2016, 65(1):169-178.

19. Halmos EP, Power VA, Shepherd SJ, Gibson PR, Muir JG: A diet low in FODMAPs reduces symptoms of irritable bowel syndrome. Gastroenterology 2014, 146(1):67-75.e65.

20. Halmos EP, Christophersen CT, Bird AR, Shepherd SJ, Gibson PR, Muir JG: Diets that differ in their FODMAP content alter the colonic luminal microenvironment. 2015, 64(1):93-100.

21. Lacy BE, Patel NK: Rome Criteria and a Diagnostic Approach to Irritable Bowel Syndrome. J Clin Med 2017, 6(11):99.

22. Francis $\mathrm{CY}$, Morris J, Whorwell PJ: The irritable bowel severity scoring system: a simple method of monitoring irritable bowel syndrome and its progress. Alimentary pharmacology \& therapeutics 1997, 11(2):395-402.

23. Association WM: World Medical Association Declaration of Helsinki: ethical principles for medical research involving human subjects. Jama 2013, 310(20):2191-2194.

24. Bacchetti De Gregoris T, Aldred N, Clare AS, Burgess JG: Improvement of phylum- and class-specific primers for real-time PCR quantification of bacterial taxa. Journal of microbiological methods 2011, 86(3):351-356.

25. Verdu EF, Armstrong D, Murray JA: Between celiac disease and irritable bowel syndrome: the "no man's land" of gluten sensitivity. The American journal of gastroenterology 2009, 104(6):1587-1594.

26. Skodje GI, Sarna VK, Minelle IH, Rolfsen KL, Muir JG, Gibson PR, Veierød MB, Henriksen C, Lundin KEA: Fructan, Rather Than Gluten, Induces Symptoms in Patients With Self-Reported Non-Celiac Gluten Sensitivity. Gastroenterology 2018, 154(3):529-539.e522.

27. McIntosh K, Reed DE, Schneider T, Dang F, Keshteli AH, De Palma G, Madsen K, Bercik P, Vanner S: FODMAPs alter symptoms and the metabolome of patients with IBS: a randomised controlled trial. Gut 2017, 66(7):1241-1251.

28. Vazquez-Roque MI, Camilleri M, Smyrk T, Murray JA, Marietta E, O'Neill J, Carlson P, Lamsam J, Janzow D, Eckert $D$ et al: A controlled trial of gluten-free diet in patients with irritable bowel syndrome-diarrhea: effects on bowel frequency and intestinal function. Gastroenterology 2013, 144(5):903-911.e903.

29. Wilson B, Rossi M, Kanno T, Hough R, Probert C, Irving P, James Mason A, Lomer M, Whelan K: PWE126 Low fodmap diet effect on IBS gastrointestinal microbiome and metabolites and prediction of response. Gut 2018, 67(Suppl 1):A181-A182. 
30. Silk DB, Davis A, Vulevic J, Tzortzis G, Gibson GR: Clinical trial: the effects of a transgalactooligosaccharide prebiotic on faecal microbiota and symptoms in irritable bowel syndrome. Alimentary pharmacology \& therapeutics 2009, 29(5):508-518.

31. Staudacher HM, Lomer MCE, Farquharson FM, Louis P, Fava F, Franciosi E, Scholz M, Tuohy KM, Lindsay JO, Irving PM et al: A Diet Low in FODMAPs Reduces Symptoms in Patients With Irritable Bowel Syndrome and A Probiotic Restores Bifidobacterium Species: A Randomized Controlled Trial. Gastroenterology 2017, 153(4):936-947.

32. Rao SS, Yu S, Fedewa A: Systematic review: dietary fibre and FODMAP-restricted diet in the management of constipation and irritable bowel syndrome. Alimentary pharmacology \& therapeutics 2015, 41(12):1256-1270.

33. Chumpitazi BP, Cope JL, Hollister EB, Tsai CM, McMeans AR, Luna RA, Versalovic J, Shulman RJ: Randomised clinical trial: gut microbiome biomarkers are associated with clinical response to a low FODMAP diet in children with the irritable bowel syndrome. Alimentary pharmacology \& therapeutics 2015, 42(4):418-427.

34. Bennet SMP, Bohn L, Storsrud S, Liljebo T, Collin L, Lindfors P, Tornblom H, Ohman L, Simren M: Multivariate modelling of faecal bacterial profiles of patients with IBS predicts responsiveness to a diet low in FODMAPs. Gut 2018, 67(5):872-881.

35. Tap J, Derrien M, Tornblom H, Brazeilles R, Cools-Portier S, Dore J, Storsrud S, Le Neve B, Ohman L, Simren M: Identification of an Intestinal Microbiota Signature Associated With Severity of Irritable Bowel Syndrome. Gastroenterology 2017, 152(1):111-123.e118.

36. Chong PP, Chin VK, Looi CY, Wong WF, Madhavan P, Yong VC: The Microbiome and Irritable Bowel Syndrome - A Review on the Pathophysiology, Current Research and Future Therapy. Front Microbiol 2019, 10(1136).

37. Chung C-S, Chang P-F, Liao C-H, Lee T-H, Chen Y, Lee Y-C, Wu M-S, Wang H-P, Ni Y-H: Differences of microbiota in small bowel and faeces between irritable bowel syndrome patients and healthy subjects. Scandinavian Journal of Gastroenterology 2016, 51(4):410-419.

38. Dior M, Delagreverie H, Duboc H, Jouet P, Coffin B, Brot L, Humbert L, Trugnan G, Seksik P, Sokol H et al: Interplay between bile acid metabolism and microbiota in irritable bowel syndrome. Neurogastroenterology and motility : the official journal of the European Gastrointestinal Motility Society 2016, 28(9):1330-1340.

39. Rangel I, Sundin J, Fuentes S, Repsilber D, de Vos WM, Brummer RJ: The relationship between faecalassociated and mucosal-associated microbiota in irritable bowel syndrome patients and healthy subjects. Alimentary pharmacology \& therapeutics 2015, 42(10):1211-1221.

40. Shepherd SJ, Parker FC, Muir JG, Gibson PR: Dietary Triggers of Abdominal Symptoms in Patients With Irritable Bowel Syndrome: Randomized Placebo-Controlled Evidence. Clinical Gastroenterology and Hepatology 2008, 6(7):765-771.

41. Biesiekierski JR, Peters SL, Newnham ED, Rosella O, Muir JG, Gibson PR: No Effects of Gluten in Patients With Self-Reported Non-Celiac Gluten Sensitivity After Dietary Reduction of Fermentable, 
Poorly Absorbed, Short-Chain Carbohydrates. Gastroenterology 2013, 145(2):320-328.e323.

42. Dieterich W, Schuppan D, Schink M, Schwappacher R, Wirtz S, Agaimy A, Neurath MF, Zopf Y: Influence of low FODMAP and gluten-free diets on disease activity and intestinal microbiota in patients with non-celiac gluten sensitivity. Clinical nutrition (Edinburgh, Scotland) 2019, 38(2):697707.

43. Rajilic-Stojanovic M, Biagi E, Heilig HG, Kajander K, Kekkonen RA, Tims S, de Vos WM: Global and deep molecular analysis of microbiota signatures in fecal samples from patients with irritable bowel syndrome. Gastroenterology 2011, 141(5):1792-1801.

44. Jeffery IB, O'Toole PW, Ohman L, Claesson MJ, Deane J, Quigley EM, Simren M: An irritable bowel syndrome subtype defined by species-specific alterations in faecal microbiota. Gut 2012, 61(7):9971006.

45. Krogius-Kurikka L, Lyra A, Malinen E, Aarnikunnas J, Tuimala J, Paulin L, Makivuokko H, Kajander K, Palva A: Microbial community analysis reveals high level phylogenetic alterations in the overall gastrointestinal microbiota of diarrhoea-predominant irritable bowel syndrome sufferers. $B M C$ gastroenterology 2009, 9:95.

46. Kassinen A, Krogius-Kurikka L, Makivuokko H, Rinttila T, Paulin L, Corander J, Malinen E, Apajalahti J, Palva A: The fecal microbiota of irritable bowel syndrome patients differs significantly from that of healthy subjects. Gastroenterology 2007, 133(1):24-33.

47. Malinen E, Krogius-Kurikka L, Lyra A, Nikkila J, Jaaskelainen A, Rinttila T, Vilpponen-Salmela T, von Wright AJ, Palva A: Association of symptoms with gastrointestinal microbiota in irritable bowel syndrome. World journal of gastroenterology 2010, 16(36):4532-4540.

48. Ponnusamy K, Choi JN, Kim J, Lee SY, Lee CH: Microbial community and metabolomic comparison of irritable bowel syndrome faeces. Journal of medical microbiology 2011, 60(Pt 6):817-827.

49. De Filippo C, Cavalieri D, Di Paola M, Ramazzotti M, Poullet JB, Massart S, Collini S, Pieraccini G, Lionetti P: Impact of diet in shaping gut microbiota revealed by a comparative study in children from Europe and rural Africa. Proceedings of the National Academy of Sciences 2010, 107(33):1469114696.

50. Kolodziejczyk AA, Zheng D, Elinav E: Diet-microbiota interactions and personalized nutrition. Nature Reviews Microbiology 2019, 17(12):742-753.

51. Bennet SMP, Ohman L, Simren M: Gut microbiota as potential orchestrators of irritable bowel syndrome. Gut Liver 2015, 9(3):318-331.

52. Rigsbee L, Agans R, Shankar V, Kenche H, Khamis HJ, Michail S, Paliy O: Quantitative profiling of gut microbiota of children with diarrhea-predominant irritable bowel syndrome. The American journal of gastroenterology 2012, 107(11):1740-1751.

53. Casen C, Vebo HC, Sekelja M, Hegge FT, Karlsson MK, Ciemniejewska E, Dzankovic S, Froyland C, Nestestog R, Engstrand $L$ et al: Deviations in human gut microbiota: a novel diagnostic test for determining dysbiosis in patients with IBS or IBD. Alimentary pharmacology \& therapeutics 2015, 42(1):71-83. 
54. Hustoft TN, Hausken T, Ystad SO, Valeur J, Brokstad K, Hatlebakk JG, Lied GA: Effects of varying dietary content of fermentable short-chain carbohydrates on symptoms, fecal microenvironment, and cytokine profiles in patients with irritable bowel syndrome. Neurogastroenterology and motility : the official journal of the European Gastrointestinal Motility Society 2017, 29(4).

55. Taverniti V, Guglielmetti S: Methodological issues in the study of intestinal microbiota in irritable bowel syndrome. World journal of gastroenterology 2014, 20(27):8821-8836.

56. Liu HN, Wu H, Chen YZ, Chen YJ, Shen XZ, Liu TT: Altered molecular signature of intestinal microbiota in irritable bowel syndrome patients compared with healthy controls: A systematic review and meta-analysis. Digestive and liver disease : official journal of the Italian Society of Gastroenterology and the Italian Association for the Study of the Liver 2017, 49(4):331-337.

57. Maccaferri S, Candela M, Turroni S, Centanni M, Severgnini M, Consolandi C, Cavina P, Brigidi P: IBSassociated phylogenetic unbalances of the intestinal microbiota are not reverted by probiotic supplementation. Gut microbes 2012, 3(5):406-413.

58. Hansen LBS, Roager HM, Sondertoft NB, Gobel RJ, Kristensen M, Valles-Colomer M, Vieira-Silva S, Ibrugger S, Lind MV, Maerkedahl RB et al: A low-gluten diet induces changes in the intestinal microbiome of healthy Danish adults. Nature communications 2018, 9(1):4630.

59. Chander AM, Yadav H, Jain S, Bhadada SK, Dhawan DK: Cross-Talk Between Gluten, Intestinal Microbiota and Intestinal Mucosa in Celiac Disease: Recent Advances and Basis of Autoimmunity. Front Microbio/ 2018, 9(2597).

60. Collado MC, Donat E, Ribes-Koninckx C, Calabuig M, Sanz Y: Imbalances in faecal and duodenal Bifidobacterium species composition in active and non-active coeliac disease. BMC Microbiology 2008, 8(1):232.

61. Golfetto L, de Senna FD, Hermes J, Beserra BT, Franca Fda S, Martinello F: Lower bifidobacteria counts in adult patients with celiac disease on a gluten-free diet. Arquivos de gastroenterologia 2014, 51(2):139-143.

62. Ghoshal UC, Shukla R, Ghoshal U, Gwee K-A, Ng SC, Quigley EMM: The gut microbiota and irritable bowel syndrome: friend or foe? Int J Inflam 2012, 2012:151085-151085.

63. De Palma G, Nadal I, Collado MC, Sanz Y: Effects of a gluten-free diet on gut microbiota and immune function in healthy adult human subjects. The British journal of nutrition 2009, 102(8):1154-1160.

64. Booijink CC, El-Aidy S, Rajilic-Stojanovic M, Heilig HG, Troost FJ, Smidt H, Kleerebezem M, De Vos WM, Zoetendal EG: High temporal and inter-individual variation detected in the human ileal microbiota. Environmental microbiology 2010, 12(12):3213-3227.

65. Jalanka-Tuovinen J, Salojarvi J, Salonen A, Immonen O, Garsed K, Kelly FM, Zaitoun A, Palva A, Spiller RC, de Vos WM: Faecal microbiota composition and host-microbe cross-talk following gastroenteritis and in postinfectious irritable bowel syndrome. Gut 2014, 63(11):1737-1745.

66. Salonen A, de Vos WM, Palva A: Gastrointestinal microbiota in irritable bowel syndrome: present state and perspectives. Microbiology (Reading, England) 2010, 156(Pt 11):3205-3215. 
67. Shulman RJ, Eakin MN, Czyzewski DI, Jarrett M, Ou C-N: Increased gastrointestinal permeability and gut inflammation in children with functional abdominal pain and irritable bowel syndrome. The Journal of pediatrics 2008, 153(5):646-650.

68. Dunlop SP, Hebden J, Campbell E, Naesdal J, Olbe L, Perkins AC, Spiller RC: Abnormal intestinal permeability in subgroups of diarrhea-predominant irritable bowel syndromes. American Journal of Gastroenterology 2006, 101(6):1288-1294.

69. Marshall J, Thabane M, Garg A, Clark W, Meddings J, Collins S, Investigators W: Intestinal permeability in patients with irritable bowel syndrome after a waterborne outbreak of acute gastroenteritis in Walkerton, Ontario. Alimentary pharmacology \& therapeutics 2004, 20(11-12):13171322.

70. Spiller R, Jenkins D, Thornley J, Hebden J, Wright T, Skinner M, Neal K: Increased rectal mucosal enteroendocrine cells, $T$ lymphocytes, and increased gut permeability following acuteCampylobacter enteritis and in post-dysenteric irritable bowel syndrome. Gut 2000, 47(6):804-811.

\section{Figures}




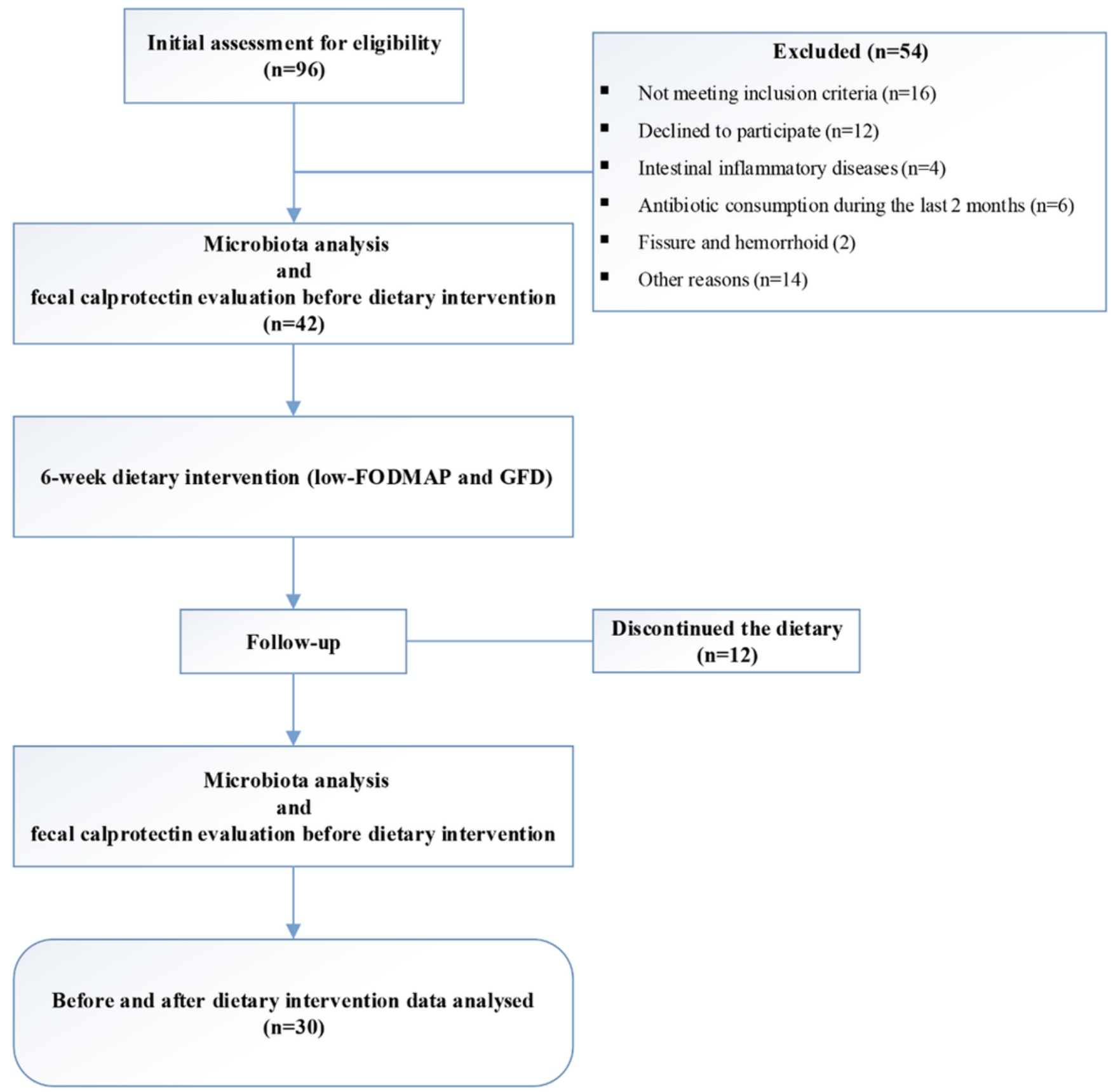

Figure 1

Schematic of recruitment process and study design. 

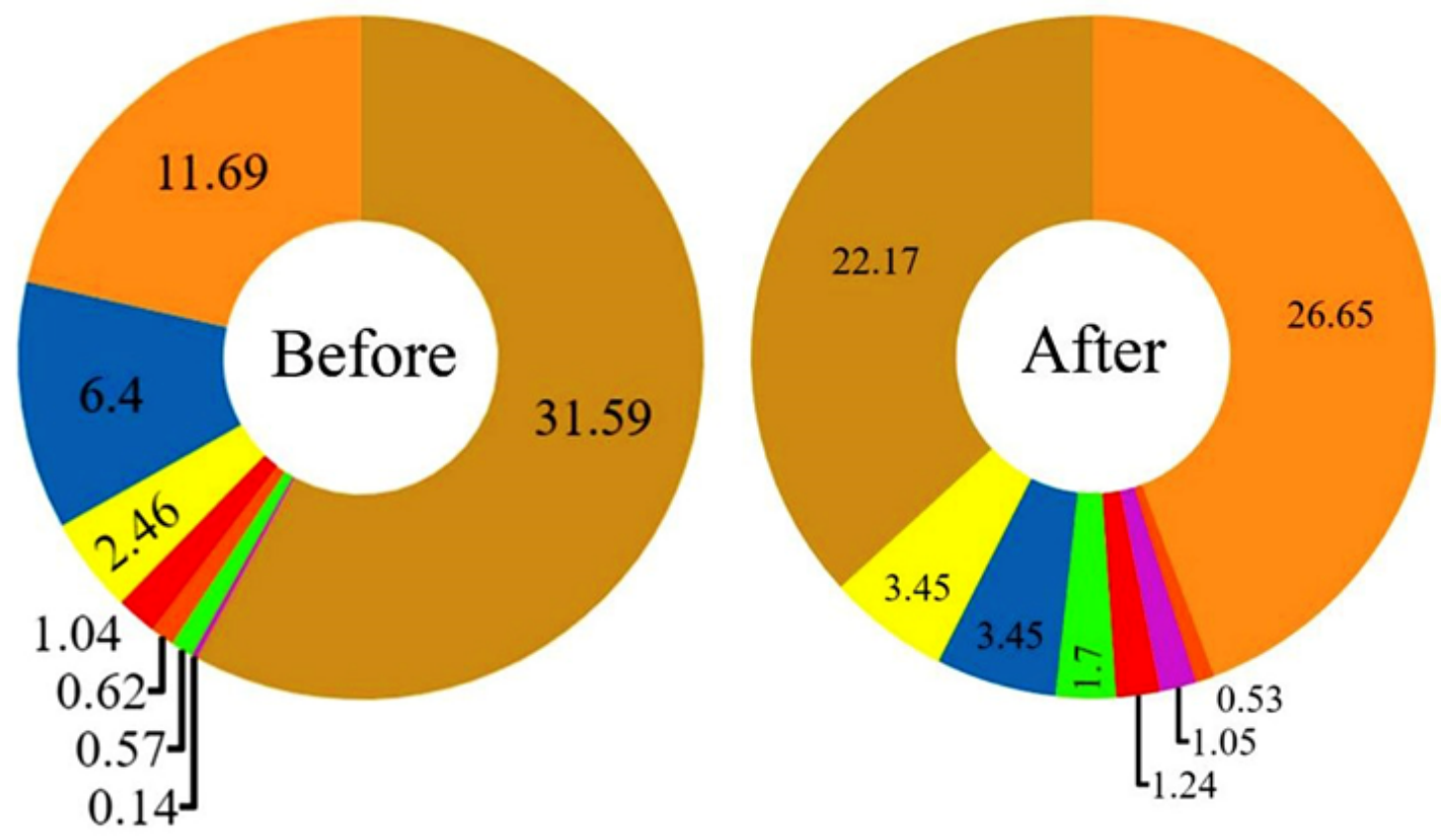

Bacteroidetes
Firmicutes
Actinobacteria
Ruminococcus
Bifidobacterium
Enterobacteriaceae
Lactobacillus
Streptococcus

Figure 2

The relative abundance and diversity of intestinal microbiota in IBS patients before and after GFD and low-FODMAP diet are illustrated in Figure 2A and 2B, respectively. Each color corresponds to a type of microbiota included in this study. 
$\mathbf{A}$

Before GFD and low-FODMAP diet

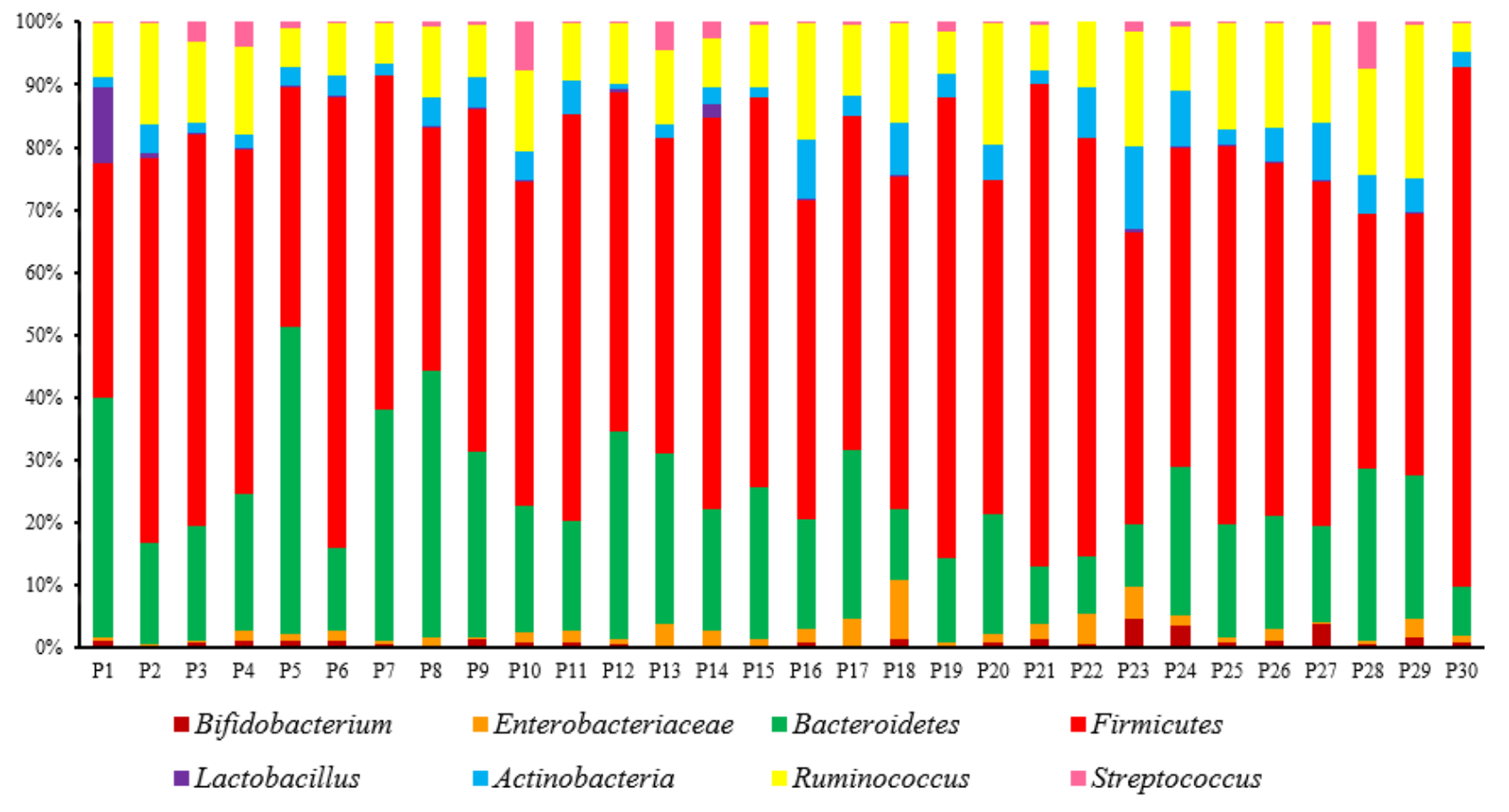

B

After GFD and low-FODMAP diet

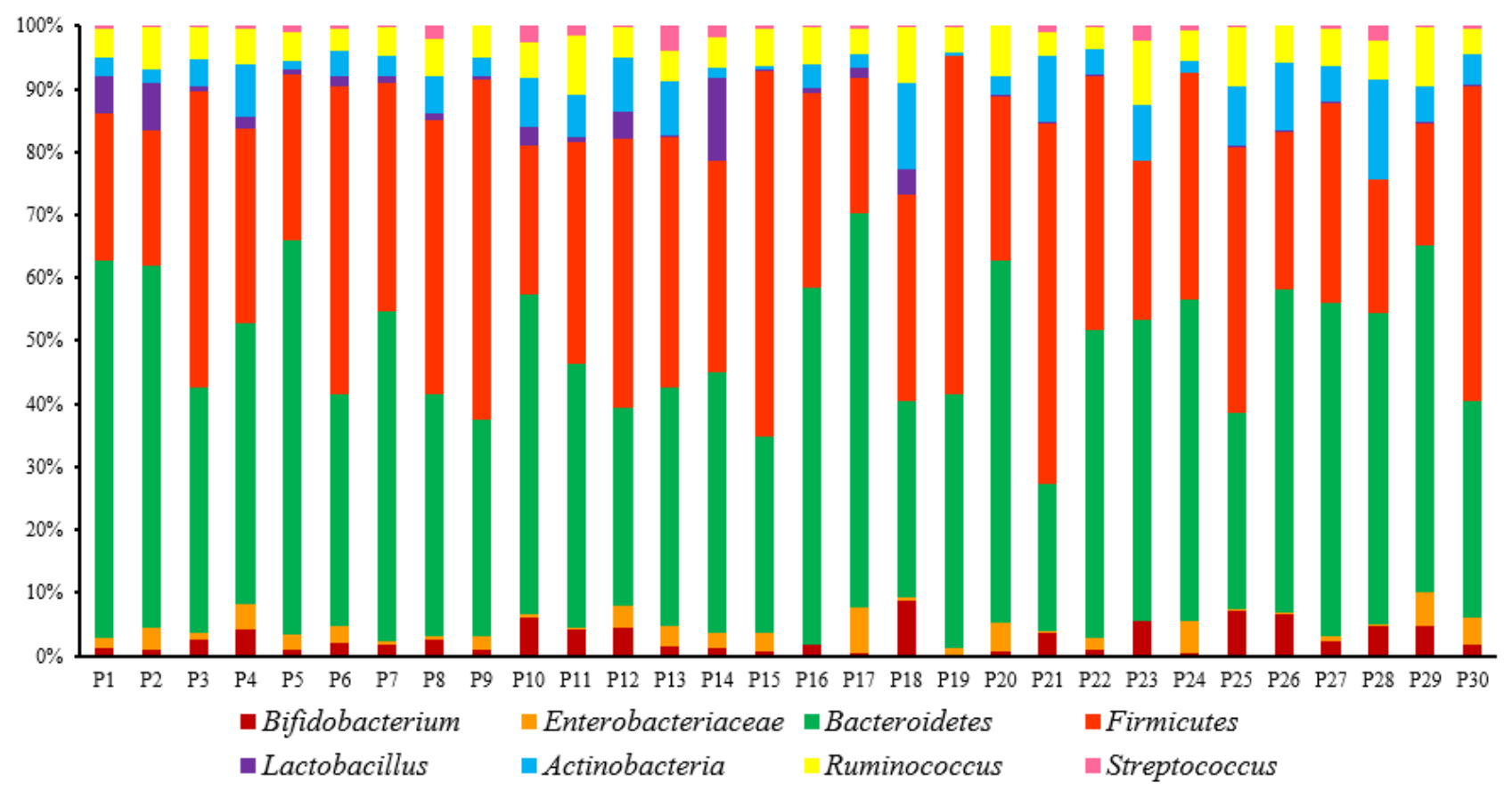

Figure 3

Pie charts representing the mean percentage of the bacterial taxa that constitute the fecal microbiota in IBS patients before and after GFD and low-FODMAP diet. 


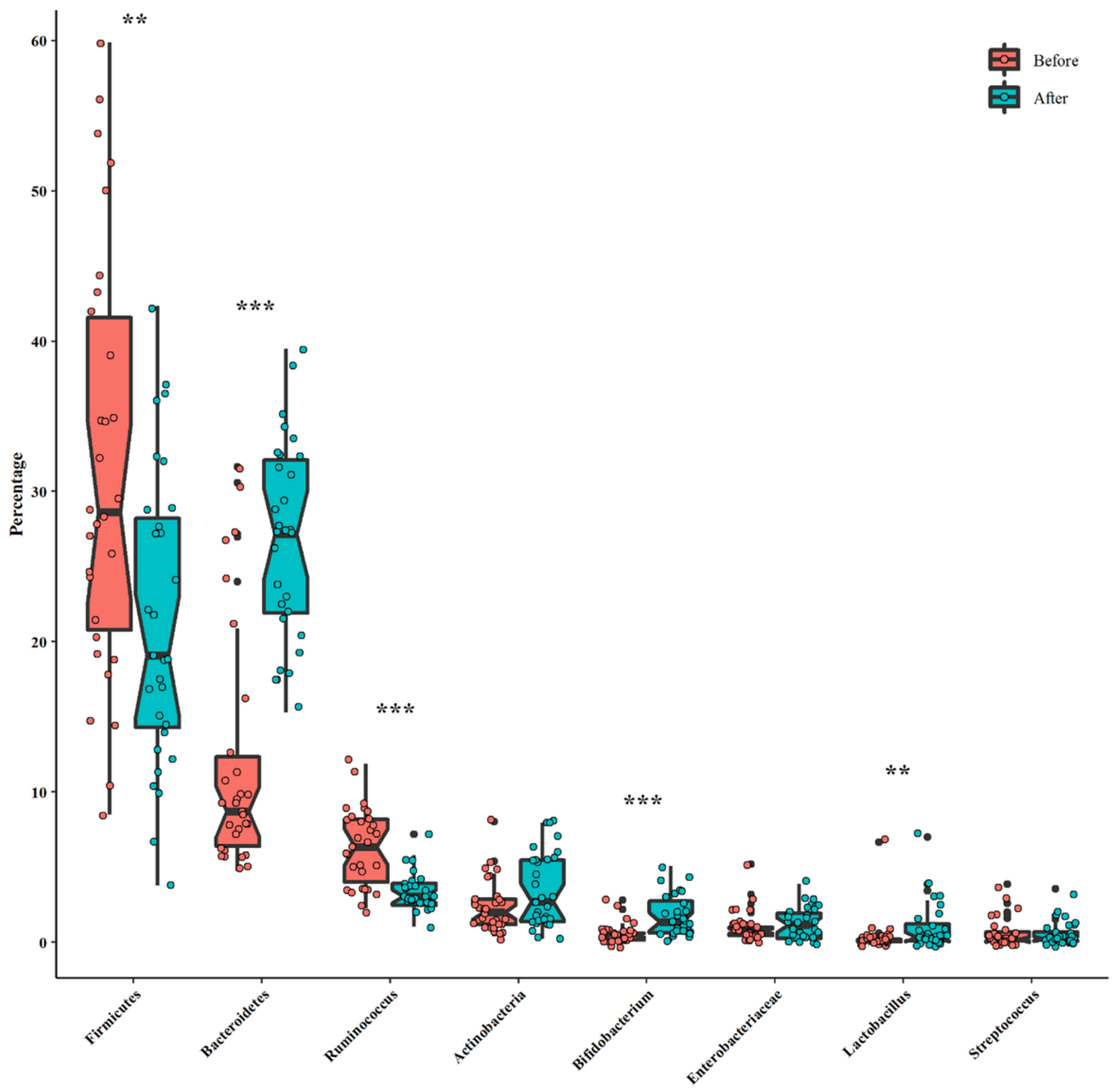

Figure 4

Box plot for the distribution of the selected bacterial taxa by median abundance that constitute the fecal microbiota in IBS patients before and after GFD and low-FODMAP diet. 


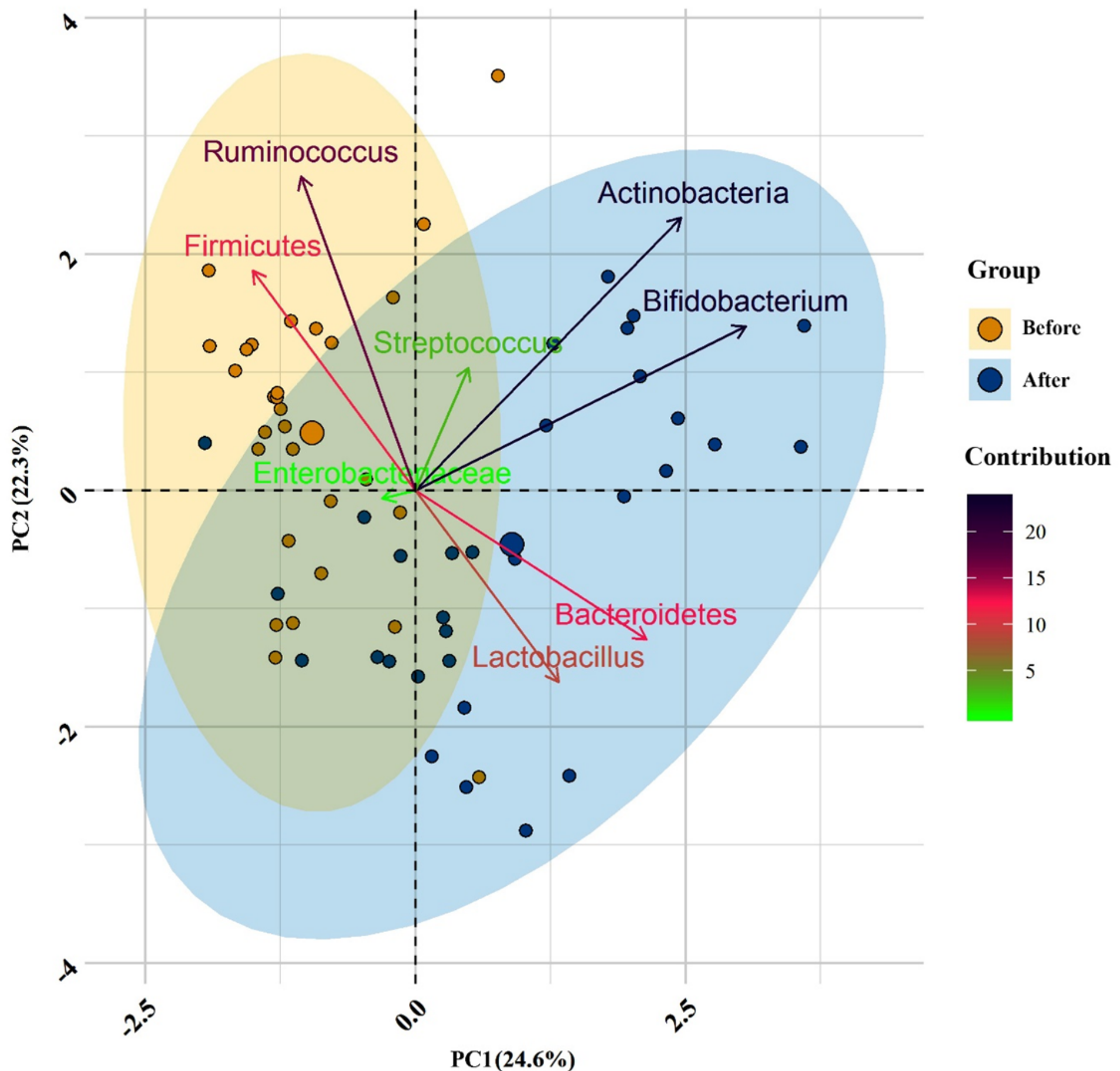

Figure 5

Bacterial community clustering using principal component analysis (PCA) before and after GFD and lowFODMAP diet in IBS patients. Percentage values in parentheses next to PC1 and PC2 represents percentage of variance explained by each component. Arrows show the contribution of each type of microbiota on the PC1 and PC2. Each data point denotes an individual patient, colored based on their group. 


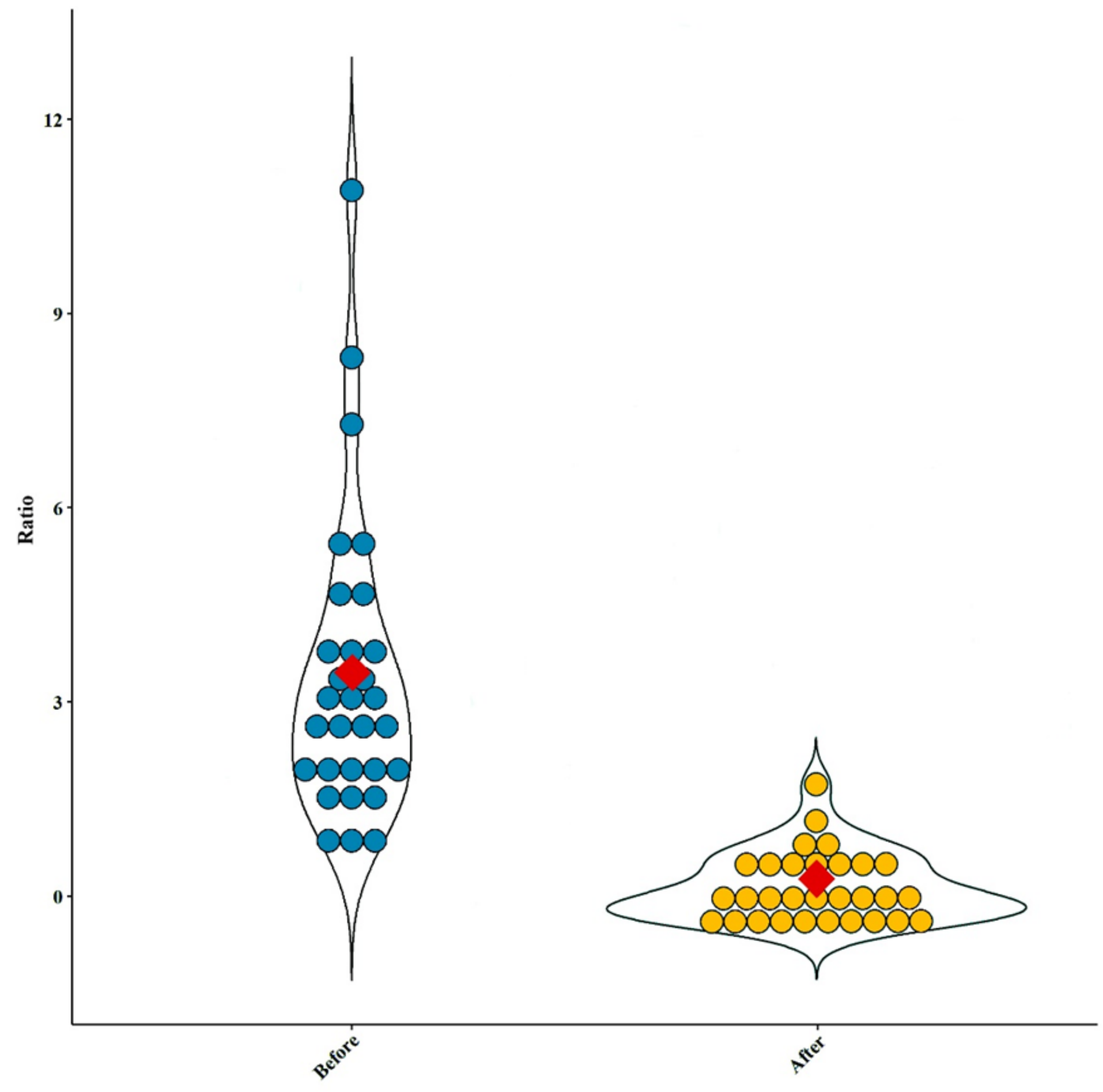

Figure 6

Violin plots showing the Firmicutes to Bacteroidetes (F/B) ratio before and after GFD and low-FODMAP diet in IBS patients. This ratio was significantly $(P=0.001)$ decreased after the dietary intervention compared with baseline. Mean values of F/B ratio are marked as the red rhombus. 


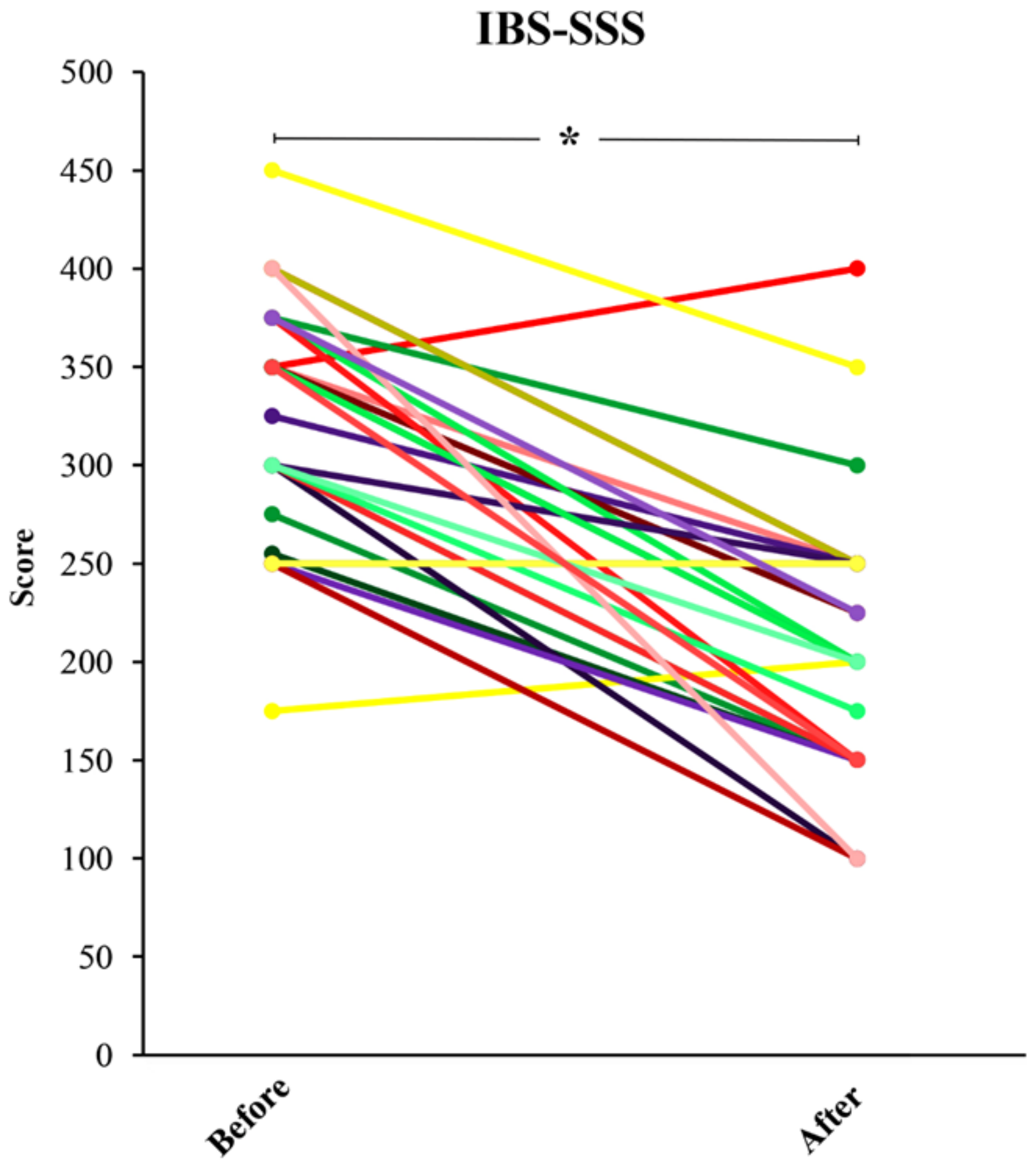

Figure 7

IBS symptom severity score (IBS-SSS) in IBS patients before and after the dietary intervention. IBS-SSS was reduced in patients after the end of the dietary intervention compared with baseline $(P=0.038)$. 


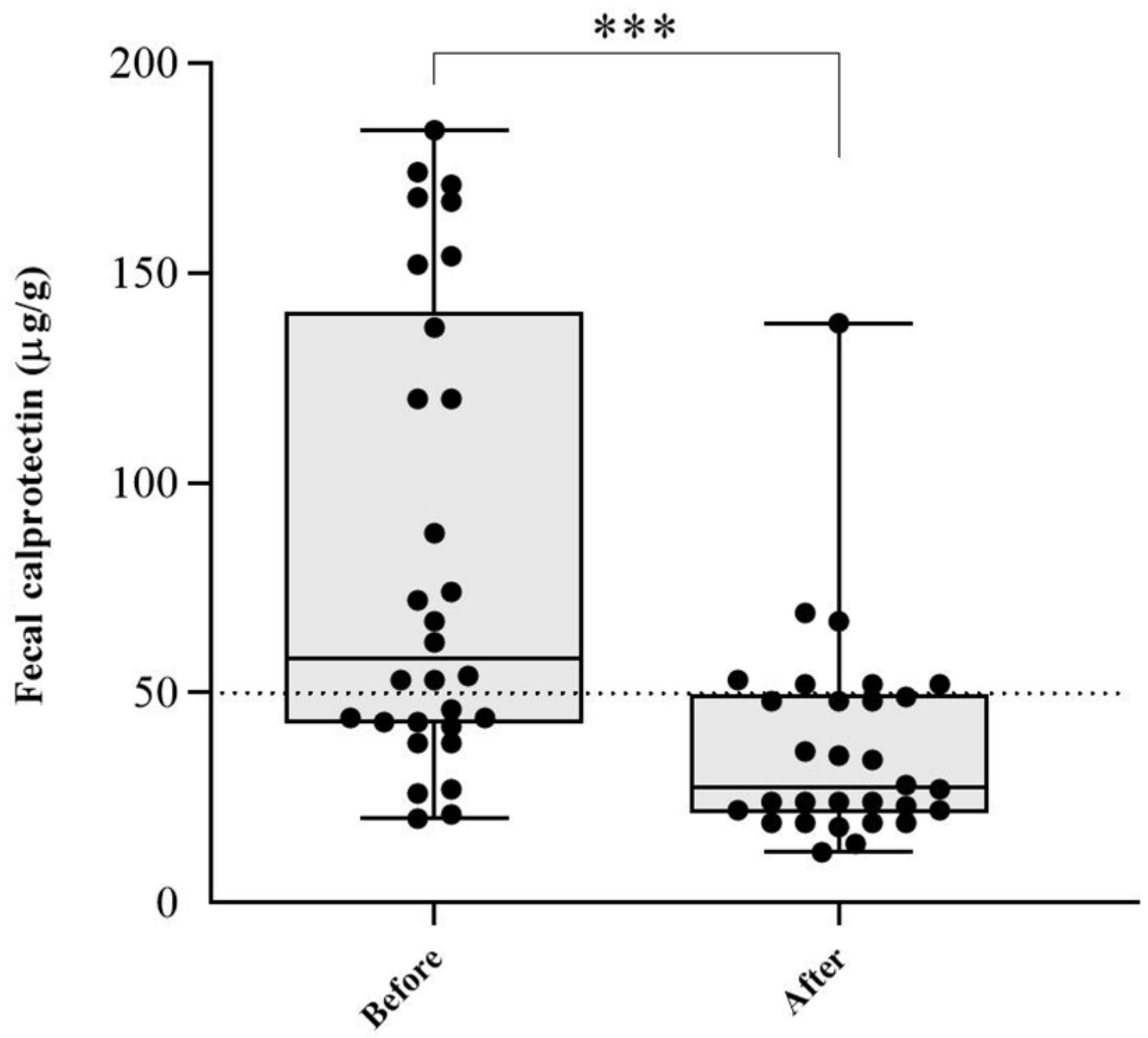

Figure 8

Values of fecal calprotectin level in IBS patients before and after a six-week of GFD and low-FODMAP diet. ${ }^{*} * \mathrm{P}<0.001$.

\section{Supplementary Files}

This is a list of supplementary files associated with this preprint. Click to download.

- GraphicalAbstract.jpg

- Tables1.docx 